\section{Oligo Design: a computer program for development of probes for oligonucleotide microarrays}

\author{
Keith E. Herold ${ }^{1,2}$ and Avraham Rasooly ${ }^{2}$ \\ ${ }^{1}$ University of Maryland, College Park and ${ }^{2}$ Food and Drug Administration, \\ Rockville, MD, USA \\ BioTechniques 35:1216-1221 (December 2003)
}

Oligonucleotide microarrays have demonstrated potential for the analysis of gene expression, genotyping, and mutational analysis. Our work focuses primarily on the detection and identification of bacteria based on known short sequences of DNA. Oligo Design, the software described here, automates several design aspects that enable the improved selection of oligonucleotides for use with microarrays for these applications. Two major features of the program are: (i) a tiling algorithm for the design of short overlapping temperature-matched oligonucleotides of variable length, which are useful for the analysis of single nucleotide polymorphisms and (ii) a set of tools for the analysis of multiple alignments of gene families and related short DNA sequences, which allow for the identification of conserved DNA sequences for PCR primer selection and variable DNA sequences for the selection of unique probes for identification. Note that the program does not address the full genome perspective but, instead, is focused on the genetic analysis of short segments of DNA. The program is Internet-enabled and includes a built-in browser and the automated ability to download sequences from GenBank ${ }^{\circledR}$ by specifying the GI number. The program also includes several utilities, including audio recital of a DNA sequence (useful for verifying sequences against a written document), a random sequence generator that provides insight into the relationship between melting temperature and GC content, and a PCR calculator.

\section{INTRODUCTION}

The use of microarrays for genotyping and mutational analysis, in the context of bacterial identification using short DNA segments, requires a carefully chosen set of oligonucleotides tethered to the array. A basic element of oligonucleotide microarray design, especially microarrays for single nucleotide polymorphism (SNP) detection and analysis of closely related DNA sequences, is the use of relatively short oligonucleotide probes (15-25 nucleotides). Discrimination of DNA sequences maximizing the instability caused by a single-base mismatch using short oligonucleotides sometimes lacks the required specificity. One approach to address this is called tiling, the use of short overlapping oligonucleotide probes (1-3) that provide high specificity through overlapping sequences. The effective use of such short probes in oligonucleotide microarrays relies on matching the melting temperature $\left(\mathrm{T}_{\mathrm{m}}\right)$ of all the probes chosen for the array for effective and uniform hybridization.

The $T_{m}$ of a DNA duplex is defined as the temperature where one-half of the nucleotides are paired, and one-half are unpaired (4). $\mathrm{T}_{\mathrm{m}}$ is typically measured as the midpoint of the spectroscopic hyperchromic absorbance shift during DNA melting. $T_{m}$ depends primarily on the DNA GC base content, cation concentration of the buffer, and the DNA double-strand length $(4,5)$. Historically, the $T_{m}$ of DNA was predicted by formulas that account for differing GC content in various buffers (6) and the length of the oligonucleotide $(4,7,8)$. Currently, the most accurate prediction of $\mathrm{T}_{\mathrm{m}}$ for oligonucleotide DNA uses the thermodynamic nearest-neighbor model (9-11). The nearest-neighbor model for nucleic acids assumes that the stability of a given base pair depends on the identity and orientation of neighboring base pairs. The basic assumption is that probe free energy can be calculated from the enthalpy and entropy of all nearest-neighbor pairs. Thermodynamic values for entropy and enthalpy of each possible matched near- est-neighbor pair have been determined (9-11) including dangling ends (12) and base mismatches (13). Nearest-neighbor calculations for $\mathrm{T}_{\mathrm{m}}$ prediction are useful for microarray (14) design and for the selection of PCR primers and hybridization probes $(15,16)$.

Another microarray application is the discrimination of closely related DNA sequences. Our approach for developing clinical microarrays for microbial identification $(17,18)$ is to choose gene families shared among microbial species to amplify by PCR using universal primers (degenerate) designed to anneal to conserved regions that straddle variable regions. The variable regions are used for microbial species identification on the microarray. To find such regions, the approach is based on multiple alignment using standard tools [e.g., ClustalX (ftp: //ftp-igbmc.u-strasbg.fr/pub/ClustalX/)] (19) to align the sequences.

Oligo Design, the program introduced here, is designed to simplify the development of primers and oligonucleotide probes for bacterial detection and identification and to provide tools for designing overlapping oligonucleotides with temperature-matched oligonucleotides (tiling).

\section{MATERIALS AND METHODS}

Oligo Design is Microsoft ${ }^{\circledR}$ Windows ${ }^{\circledR}$-based (XP 2000) and is written in Visual Basic ${ }^{\mathrm{TM}}$. It incorporates a graphical user interface, is Internet-enabled (automatic connection to GenBank), and has on-line help. The program was developed based on a combination of laboratory experience and the literature on microarray and PCR methods. The program supports our ongoing research on bacterial identification and is constantly expanding as new needs are identified. We have performed continuous verification of the output of the program in support of active research. The current version of the program, version 1.3 , is available free of charge at http://www.enme.umd.edu/ bioengineering/ and at the Software $\mathrm{Li}$ brary at http://www.BioTechniques.com.

\section{Melting Temperature Calculations}

The $T_{m}$ calculations are based on the nearest-neighbor thermodynamic 
model $(10,11,20)$, which is represented by

$$
T_{m}=\frac{\Delta H}{\Delta S+R \ln C_{t}}+16.6 \log _{10}\left[x_{\text {salt }}\right]
$$

where

$$
\Delta H=\sum_{i} n_{i} \Delta H_{i}+\Delta H_{i n i t}+\Delta H_{i n i t}+\Delta H_{\text {sym }}
$$

$$
\Delta S=\sum_{i} n_{i} \Delta S_{i}+\Delta S_{i n i t}+\Delta S_{i n i t}+\Delta S_{\text {sym }}
$$

and where $\Delta H_{i}$ and $\Delta S_{i}$ are the enthalpy and entropy terms associated with the $n-1$ pairs of neighboring nucleotides contained in the sequence of interest. $R$ is the universal gas constant $(1.987 \mathrm{Cal} / \mathrm{mol}$ - $\mathrm{K}) . C_{t}$ is the total molar concentration of single strands when oligonucleotides are self complementary or it is equal to one-fourth of this concentration when sequences are not self complementary (10). The model assumes an equal concentration of the plus and minus strands. The program allows the user to input values for salt and oligonucleotide concentrations. The nearest-neighbor model is based on data for hybridization in free solution and thus must be used with some caution for predicting hybridization on a solid-surface microarray. One way to utilize the model for microarray analysis in lieu of solid-phase data is to assume that the relative stability found in free solution will be reproduced in hybridization on a surface.

Each of the nearest-neighbor papers cited proposes a different set of constants for the nearest-neighbor model. The program allows for any of these formulations to be selected from a pull-down menu. In addition to the nearest-neighbor models, two simplified models based on GC content are provided for reference. Note that these simplified models have substantial limitations compared to the nearestneighbor model.

In addition to the standard $\mathrm{T}_{\mathrm{m}}$ predictions for the denaturation of double-stranded Watson-Crick base paired DNA, the program includes a nearest-neighbor model for single-base mismatches $(13,21-24)$. This model is quite useful for designing microarrays for SNP detection.

The $T_{m}$ models allow the user to estimate the effects of various parameters on the microarray design. These tools provide significant insight over older methods that depended largely on the GC content to estimate $T_{m}$. The program includes a tool designed to provide insight into the correlation between GC content and $\mathrm{T}_{\mathrm{m}}$. This tool generates a large random set of sequences of a particular length and computes both $\mathrm{T}_{\mathrm{m}}$ and the GC content for each sequence (Figure 1). The $\mathrm{T}_{\mathrm{m}}$ is plotted in three complementary formats: (i) total number of oligonucleotides versus $\mathrm{T}_{\mathrm{m}}$; (ii) $\mathrm{T}_{\mathrm{m}}$ versus the $\mathrm{GC}$ content; and (iii) number of oligonucleotides versus $T_{m}$ at three particular GC fractions. The spread of the resulting data provides a very informative view of the influence of GC content, oligonucleotide length, and sequence on the $T_{m}$.

\section{Tiling Algorithm}

Tiling is the use of overlapping oligonucleotide probes. Although tiling may be used for the optimal coverage of high-complexity DNA (25), our program is designed for the analysis of short oligonucleotide microarrays. To develop a tiled microarray for a specific gene, the user enters a target sequence for analysis using one of several input schemes (e.g., keyboard entry, cut and paste, or import from GenBank). After entry, the program scans the input sequence and computes the average $T_{m}$ for the user-selected oligonucleotide length to provide guidance for a reasonable target temperature. For tiling, the program steps through the input sequence and finds the oligonucleotide at each base position that most closely matches the target $T_{m}$. The result of this initial pass is an array containing

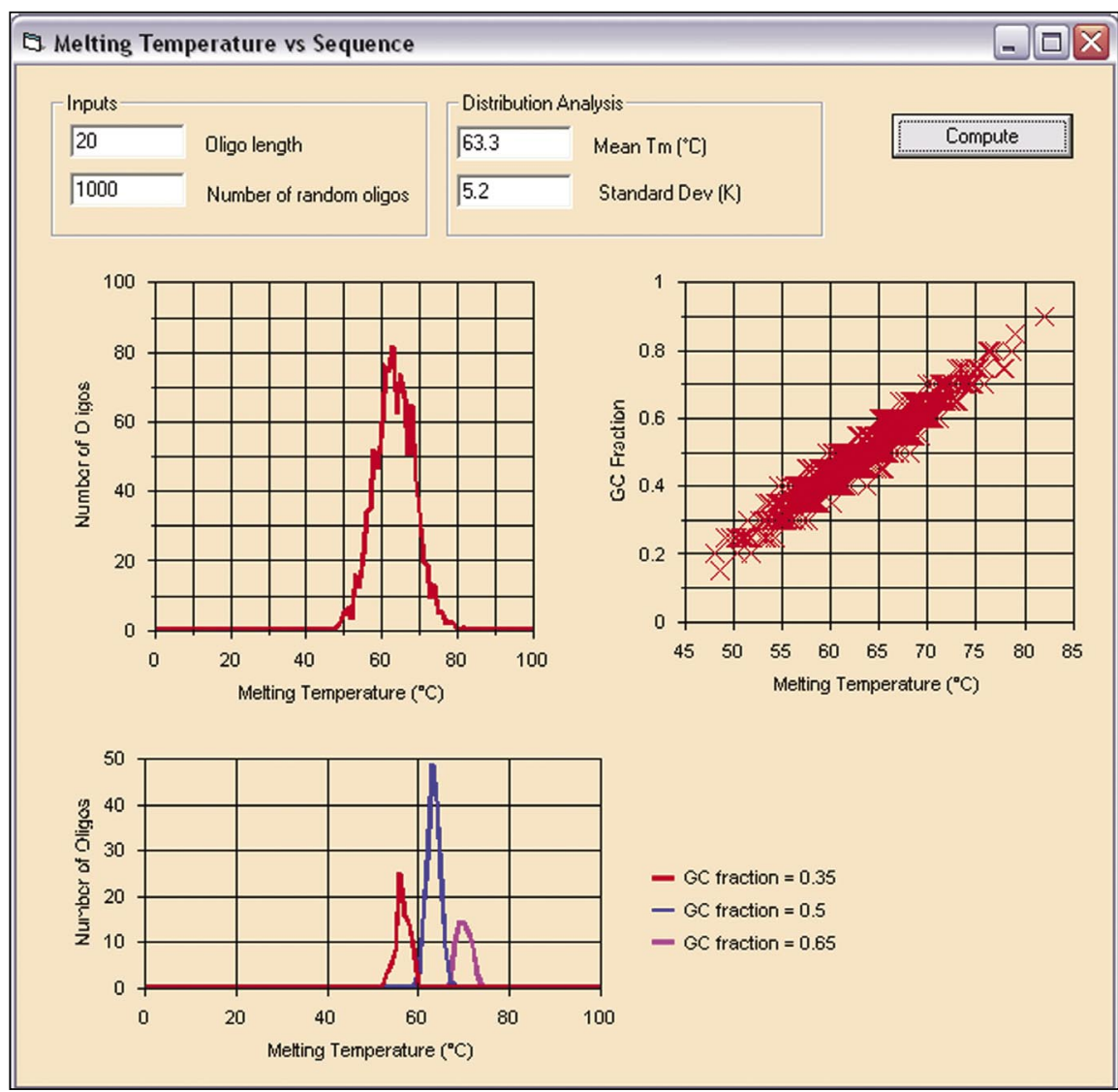

Figure 1. Correlation between GC content and melting temperature $\left(\mathbf{T}_{\mathbf{m}}\right)$. Plot showing the correlation between GC content and $T_{m}$ of a large random set of sequences of a particular length. The $T_{m}$ is plotted in three complementary formats: (i) the total number of oligonucleotides versus $\mathrm{T}_{\mathrm{m}}$; (ii) $\mathrm{T}_{\mathrm{m}}$ versus the GC content; and (iii) number of oligonucleotides versus $\mathrm{T}_{\mathrm{m}}$ at three particular $\mathrm{GC}$ fractions. The spread of the resulting data shows the influences of GC content and oligonucleotide length and sequence on the $\mathrm{T}_{\mathrm{m}}$. Oligo, oligonucleotide. 
the length of the temperature-matched oligonucleotide at each base position. The results of this calculation are plotted to ensure that the target $T_{m}$ and the oligonucleotide length range are reasonable for the input sequence.

The tiling calculation uses the data from the temperature-matched oligonucleotide calculation. The inputs to the tiling calculation are the nominal base offset and the allowable range in $T_{m}$. The program steps through the temperature-matched oligonucleotide set using a step size equal to the nominal offset. At each step, the program searches in that vicinity to find the oligonucleotide that most closely matches the target $\mathrm{T}_{\mathrm{m}}$ and that meets the length range requirements. Once the program finds an oligonucleotide that meets the criteria, the program steps to the next position. If none of the oligonucleotides meet the criterion, then the program selects the one that comes closest and marks the error. The oligonucleotides resulting from the tiling calculation are displayed in a table along with various statistics. The results can also be printed and/or stored to a file for subsequent editing or direct transmittal to an oligonucleotide supplier. The results of the tiling calculation can be plotted in several formats that show the depth of redundant coverage at each base position.

As an example, a partial sequence for the 16S rRNA for Rhodococcus sp. was retrieved from the Internet by entering the GI number (27901578) in the Get Sequence window. This particular sequence is $1460 \mathrm{bp}$. When the sequence is initially downloaded, the FASTA header is included in the file. This can

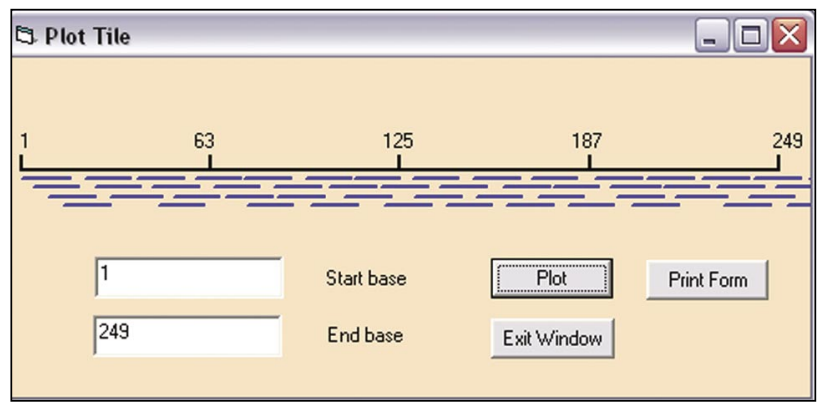

Figure 2. Tiling window of overlapping oligonucleotides. Plot showing the tiling of a 250-bp region for the 16S rRNA of Rhodococcus sp. (GI no. 27901578). The oligonucleotide ranges 10-15 bp, and the average melting temperature $\left(\mathrm{T}_{\mathrm{m}}\right)$ is $41.8^{\circ} \mathrm{C}$. The tiling window shows a set of overlapping oligonucleotides that provide redundant coverage of the sequence. Individual oligonucleotides can be distinguished on the plot. be removed by double clicking on the main text window. This sequence has a GC content of $58 \%$, as shown in the sequence statistics in the main window. For an oligonucleotide ranging 15-25 bp, the average $\mathrm{T}_{\mathrm{m}}$ is $58.2^{\circ} \mathrm{C}$.

The tiling calculation is initiated by selecting Tiling from the Analysis menu. This initiates the temperaturematched oligonucleotide calculation that takes several seconds, during which a progress monitor is displayed. When this preliminary calculation is complete, the tiling window is displayed. The tiling calculation results in a set of overlapping oligonucleotides that provides redundant coverage of the entire sequence. A plot of the coverage can be requested (Figure 2). In this case, a 249-bp region is plotted so that the individual oligonucleotides can be distinguished on the plot.

\section{SNP Analysis}

A growing range of SNP-detecting technologies based on microarrays can be found in the literature, such as minisequencing, solid-phase primer extension, and solid-phase PCR (26). Because each of these technologies depends on DNA hybridization, the program described here is a useful tool for design and analysis.

The user can enter expected SNP locations via keyboard or file input. For multiple SNPs, file input is the most convenient. The program expects a simple input file format in which each SNP location is specified on one line. For each SNP location, the program finds a temperature-matched oligonucleotide that is straddling the SNP. Four such oligonucleotides are reported for each SNP location, with one for each possible base pair at that location. For file input, all of the SNP locations are analyzed, and the output is stored in a file with a name derived from the input file. This file can be examined and edited by opening a text editor from within the program. The results from the SNP analysis can be appended to the results from the main tiling calculation to arrive at a complete design document for the array.

The program provides a calculation of the $T_{m}$ of each of the SNP-detecting oligonucleotides with a mismatch at the SNP position. This allows the user to estimate the sensitivity of the resulting array to distinguish between the various forms expected for the SNP. The user can readily investigate the influence of oligonucleotide length on the mismatch temperature difference.

\section{Multiple Sequence File Analysis}

A second major analysis feature of Oligo Design addresses the task of identifying and differentiating species and strains of bacteria on a microarray. This task is accomplished by first performing a multiple alignment on the various sequences of interest. We use ClustalX for this task (19). The output of ClustalX is a multiple sequence file (MSF) that contains the aligned sequences. We perform two different types of tasks on the MSF file: (i) find conserved regions that allow a single set of degenerate primers to amplify all sequences of interest (universal primers) and (ii) within the region straddled by the primers, find unique signatures for each of the sequences. The first step in this analysis is to read an MSF file into the program. Once it is read in, a summary showing the number of sequences and the number of bases in the alignment is produced.

Find primers. The program analyzes the MSF and assigns a score to each base position within the alignment that represents the variation among sequences at that position. That score is then used to score oligonucleotides, of length input by the user, at each base position within the file. The resulting score represents the degree of identity between the given sequences and provides a good guide as to which primers can be chosen. The program reports degenerate primers using the International Union of Pure and Applied Chemistry (IUPAC) (London, UK) base designation convention. It computes the degeneracy and gives an option to insert inosine. The conserved regions are identified for both forward 
and reverse primers, and the results are plotted and tabulated. The tabulated results can be printed or stored in a file.

Features in the primer selection algorithm include the ability to specify an exact match of a user-input number of bases at the $3^{\prime}$ end. At the $5^{\prime}$ end, the inputs can be set to ignore sequence differences that might significantly increase primer degeneracy while having little effect on the ability of the primers to anneal to the target sequences. These features can be adjusted based on user experience.

The most promising primers, both forward and reverse, are plotted and tabulated along with statistics for each. Depending on the particular set of sequences and the user inputs, this may result in a large number of promising primers. An example of the program output for a group of Staphylococcal enterotoxin genes is shown in Figure 3 , where approximately 10 forward and 10 reverse universal primer locations are identified. The $T_{m}$ reported in the primer selection table assumes
Watson-Crick annealing; for degenerate primers with some mismatch, this value will be the upper-limit $T_{m}$. The optimum hybridization temperature with degenerate primers must be determined empirically with the upper limit as a guide.

Find unique sequences. Once the primers have been chosen, then the task changes from finding conserved regions to finding sequences unique to each species that can be used for identification. The search is focused on the variable region between the flanking primers. The program automates this tedious task. For the enterotoxin example, 20 of 22 sequences can be clearly differentiated when the most widely spaced primer selection is chosen (data not shown). The ability to find unique sequences depends strongly on the particular sequences, the length of the amplified region, and the length of the probes. The $T_{m}$ of each of these probes is reported, providing the user with insight into the expected relative hybridization efficiency.

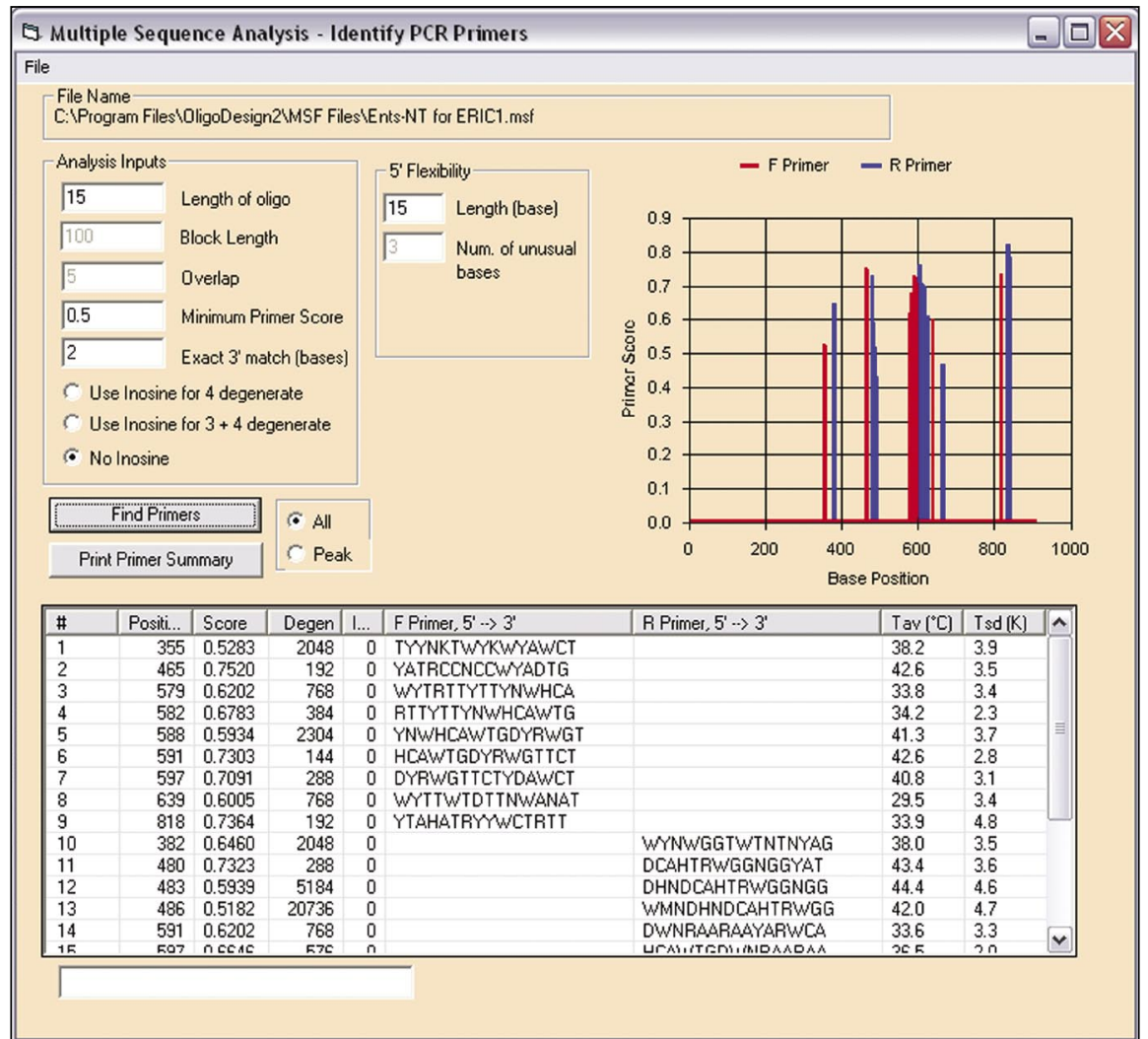

Figure 3. Multiple sequence alignment PCR primer analysis. Output of PCR primer selection for Staphylococcal enterotoxins. Approximately 10 forward and 10 reverse universal primers for the amplification of Staphylococcal enterotoxins were identified.

\section{Additional Tools}

In addition to the major tools described above, the program includes several minor tools, such as a PCR calculator and an audio sequence recital feature. The calculator window for determining PCR constituents is available from the Analysis menu. Although this is a simple calculation, it becomes tedious when multiple reactions are being prepared. The user inputs the total reaction volume and the stock and final concentrations of all the reactants, and the program calculates the volume of each reactant and the volume of the water.

Another useful feature is the audio sequence recital. This feature has been useful for verifying sequences between the hard copy and screen. The audio feature allows the user to focus on the hard copy, while the computer steps through the sequence. The user can specify the speed of recital over a range.

\section{Internet Features}

The program includes two Internet features. One is a built-in browser connected to Entrez that pops up and allows the user to search the entire PubMed and GenBank resource. If the user finds a sequence that they wish to analyze locally, the sequence can be copied and pasted into the text window in Oligo Design. This browser feature is useful for searching for unknown sequences and for allowing flexibility to search for related literature.

If the user already knows the sequence of interest (i.e., the GI number), then the second Internet feature allows single-click downloading of that sequence into the text window of the program. The sequence is downloaded in FASTA format, and the program includes a feature for stripping the FASTA header line and searching through the sequence for unexpected characters.

\section{Future Work}

Oligo Design is written as a set of supporting tools for our work on microarray-based bacterial identification. The program is under active develop- 
ment as new features are identified constantly. By making the program publicly available, we hope to expand the user base and obtain user feedback that will stimulate further refinement.

\section{ACKNOWLEDGMENTS}

This work was supported in part by the United States Department of Agriculture grant no. 0013000.

\section{REFERENCES}

1.Hacia, J.G., J.B. Fan, O. Ryder, L. Jin, K. Edgemon, G. Ghandour, R.A. Mayer, B. Sun, et al. 1999. Determination of ancestral alleles for human single-nucleotide polymorphisms using high-density oligonucleotide arrays. Nat. Genet. 22:164-167.

2.Kallioniemi, O.P. 2001. Biochip technologies in cancer research. Ann. Med. 33:142-147.

3.Cutler, D.J., M.E. Zwick, M.M. Carrasquillo, C.T. Yohn, K.P. Tobin, C. Kashuk, D.J. Mathews, N.A. Shah, et al. 2001. Highthroughput variation detection and genotyping using microarrays. Genome Res. 11:19131925 .

4.Wetmur, J.G. 1991. DNA probes: applications of the principles of nucleic acid hybridization. Crit. Rev. Biochem. Mol. Biol. 26:227-259.

5.Hall, T.J., J.W. Grula, E.H. Davidson, and R.J. Britten. 1980. Evolution of sea urchin non-repetitive DNA. J. Mol. Evol. 16:95-110.

6.Schildkraut, C. 1965. Dependence of the melting temperature of DNA on salt concentration. Biopolymers 3:195-208.

7.Wahl, G.M., S.L. Berger, and A.R. Kimmel. 1987. Molecular hybridization of immobilized nucleic acids: theoretical concepts and practical considerations. Methods Enzymol. 152: 399-407.

8.Britten, R.J., D.E. Graham, and B.R. Neufeld. 1974. Analysis of repeating DNA sequences by reassociation. Methods Enzymol. 29:363-418.

9.Sugimoto, N., S. Nakano, M. Katoh, A. Matsumura, H. Nakamuta, T. Ohmichi, M. Yoneyama, and M. Sasaki. 1995. Thermodynamic parameters to predict stability of RNA/DNA hybrid duplexes. Biochemistry 34 : 11211-11216.

10.SantaLucia, J., Jr. 1998. A unified view of polymer, dumbbell, and oligonucleotide DNA nearest-neighbor thermodynamics. Proc. Natl. Acad. Sci. USA 95:1460-1465.

11.Breslauer, K.J., R. Frank, H. Blocker, and L.A. Marky. 1986. Predicting DNA duplex stability from the base sequence. Proc. Natl. Acad. Sci. USA 83:3746-3750.

12.Bommarito, S., N. Peyret, and J. SantaLucia, Jr. 2000. Thermodynamic parameters for DNA sequences with dangling ends. Nucleic Acids Res. 28:1929-1934.

13.Allawi, H.T. and J. SantaLucia, Jr. 1998. Nearest neighbor thermodynamic parameters for internal G.A mismatches in DNA. Biochemistry $37: 2170-2179$.
14.Fotin, A., A. Drobyshev, D. Proudnikov, A. Perov, and A. Mirzabekov. 1998. Parallel thermodynamic analysis of duplexes on oligodeoxyribonucleotide microchips. Nucleic Acids Res. 26:1515-1521.

15.Rychlik, W. and R.E. Rhoads. 1989. A computer program for choosing optimal oligonucleotides for filter hybridization, sequencing and in vitro amplification of DNA. Nucleic Acids Res. 17:8543-8551.

16.Chen, H. and G. Zhu. 1997. Computer program for calculating the melting temperature of degenerate oligonucleotides used in PCR or hybridization. BioTechniques 22:1158-1160.

17.Chizhikov, V., A. Rasooly, K. Chumakov, and D.D. Levy. 2001. Microarray analysis of microbial virulence factors. Appl. Environ. Microbiol. 67:3258-3263.

18.Volokhov, D., A. Rasooly, K. Chumakov, and V. Chizhikov. 2002. Identification of Listeria species by microarray-based assay. J. Clin. Microbiol. 40:4720-4728.

19.Thompson, J.D., D.G. Higgins, and T.J. Gibson. 1994. CLUSTAL W: improving the sensitivity of progressive multiple sequence alignment through sequence weighting, position-specific gap penalties and weight matrix choice. Nucleic Acids Res. 22:4673-4680.

20.Sugimoto, N., S. Nakano, M. Yoneyama, and K. Honda. 1996. Improved thermodynamic parameters and helix initiation factor to predict stability of DNA duplexes. Nucleic Acids Res. 24:4501-4505.

21.Allawi, H.T. and J. SantaLucia, Jr. 1997. Thermodynamics and NMR of internal G.T mismatches in DNA. Biochemistry 36:1058110594.

22.Allawi, H.T. and J. SantaLucia, Jr. 1998. NMR solution structure of a DNA dodecamer containing single $G^{*} T$ mismatches. Nucleic Acids Res. 26:4925-4934.

23.Allawi, H.T. and J. SantaLucia, Jr. 1998. Nearest-neighbor thermodynamics of internal A.C mismatches in DNA: sequence dependence and pH effects. Biochemistry 37:9435-9444.

24.Allawi, H.T. and J. SantaLucia, Jr. 1998. Thermodynamics of internal C.T mismatches in DNA. Nucleic Acids Res. 26:2694-2701.

25.Berman, P., P. Bertone, B. DasGupta, M. Gerstein, M.-Y. Kao, and M. Snyder. 2002. Fast optimum genome tiling with applications to microarray design and homology search. Proceedings of the 2nd International Workshop on Algorithms in Bioinformatics. Springer-Verlag LNCS 2452:419-433.

26.Huber, M., D. Losert, R. Hiller, C. Harwanegg, M.W. Mueller, and W.M. Schmidt. 2001. Detection of single base alterations in genomic DNA by solid phase polymerase chain reaction on oligonucleotide microarrays. Anal. Biochem. 299:24-30.

Received 22 July 2003; accepted 15 September 2003.

Address correspondence to Keith E. Herold, Department of Mechanical Engineering, University of Maryland, College Park, MD 20742,USA.e-mail:herold@umd.edu 